\title{
Reactive Oxygen Species and Nuclear Factor Erythroid 2-Related Factor 2 Activation in Diabetic Nephropathy: A Hidden Target
}

Shaaban Abdo, Shao-Ling Zhang and John S.D. Chan*

Department of Medicine, University of Montreal and Research Center Hospital of QC, Canada

${ }^{*}$ Corresponding author: John S.D. Chan, Department of Medicine, University of Montreal and Research Center Hospital of the University of Montreal (CRCHUM)-Tour Viger900 Saint Denis Street, Montreal, QC, Canada H2X 0A9, Tel: (15080) 5148908000; E-mail: john.chan@umontreal.ca

Rec date: Apr 15, 2015, Acc date: May 06, 2015, Pub date: May 10, 2015

Copyright: $\odot 2015$ Abdo S et al., This is an open-access article distributed under the terms of the Creative Commons Attribution License, which permits unrestricted use, distribution, and reproduction in any medium, provided the original author and source are credited.

\begin{abstract}
Hyperglycemia, oxidative stress and renin-angiotensin system (RAS) dysfunction have been implicated in diabetic nephropathy (DN) progression, but the underlying molecular mechanisms are far from being fully understood. In addition to the systemic RAS, the existence of a local intrarenal RAS in renal proximal tubular cells has been recognized. Angiotensinogen is the sole precursor of all angiotensins (Ang). Intrarenal reactive oxygen species (ROS) generation, Ang II level and RAS gene expression are up-regulated in diabetes, indicating that intrarenal ROS and RAS activation play an important role in DN. The nuclear factor erythroid 2-related factor 2 (Nrf2)-Kelchlike $\mathrm{ECH}$-associated protein 1 (Keap1) pathway is one of the major protective processes that occurs in response to intracellular oxidative stress. Nrf2 stimulates an array of antioxidant enzymes that convert excessive ROS to less reactive or less damaging forms. Recent studies have, however, revealed that Nrf2 activation might have other undesirable effects in diabetic animals and in diabetic patients with chronic kidney disease. This mini-review summarizes current knowledge of the relationship between ROS, Nrf2 and intra renal RAS activation in DN progression as well as possible novel target(s) for DN treatment.
\end{abstract}

Keywords: Nrf2; Angiotensinogen expression; Renin-angiotensin system

\begin{abstract}
Abbreviations:
ACE: Angiotensin-Converting Enzyme; Agt: Angiotensinogen; Ang II: Angiotensin II; Ang 1-7: Angiotensin 1-7; ARE: Antioxidant Responsive Element; AT1R: Ang II subtype 1 receptor; AT2R: Ang II subtype 2 receptor; BP: Blood Pressure; Cat: Catalase; CKD: Chronic Kidney Disease; DN: Diabetic Nephropathy; ESRD: End Stage Renal Disease; GFR: Glomerular Filtration Rate; HG: High Glucose; hnRNP $\mathrm{F}$ and hnRNP K: Heterogeneous Nuclear Ribonucleoproteins F and K; H2O2: Hydrogen Peroxide; Keap1: Kelch-like ECH-associated Protein 1; NADPH oxidase: Nicotinamide Adenine Dinucleotide Phosphate Oxidase; Nrf2: Nuclear Factor Erythroid 2-Related Factor 2; O2•-: Superoxide; RAS: Renin-Angiotensin System; Res: Responsive Elements; ROS: Reactive Oxygen Species; RPTs: Renal Proximal Tubules; Runx2: Runt-Related Transcription Factor 2; RPTCs: Renal Proximal Tubular Cells; STZ: Streptozotocin; T1D and T2D: Type 1 And Type 2 Diabetes Mellitus; ZDF: zucker diabetic fatty rats
\end{abstract}

\section{Introduction}

Diabetes is an epidemic disease that is imposing a heavy healthcare burden globally. Its incidence is continuing to rise unabated. According to recent estimates by the International Diabetes Federation, the number of people with diabetes will surge from 382 to 592 million in less than 25 years. Diabetes and its associated complications caused 5.1 million deaths in 2013 [1]. Diabetic nephropathy (DN), a clinical syndrome, is the result of glucolipotoxicity. It impacts the kidneys, eliciting progressive renal insufficiency, persistent albuminuria, hypertension and decreased glomerular filtration rate (GFR) [2]. DN affects approximately one- third of people with type 1 or type 2 diabetes mellitus (T1D and T2D respectively) [3]. It is the most common cause of end-stage renal disease (ESRD) in the West and possibly throughout the world, accounting for more than $50 \%$ of all patients with ESRD. Although insulin and oral anti-diabetic drugs, along with diet and exercise, are the cornerstones of diabetes mellitus and DN treatment [4,5], their underlying mechanisms remain incompletely understood.

\section{The Renin-Angiotensin System (RAS)}

The RAS is a hormonal system that regulates sodium balance, body fluid homeostasis and arterial pressure [6]. All RAS components have been identified in the kidneys [7], including mRNA and protein of angiotensinogen (Agt), renin, angiotensin-converting enzyme (ACE), angiotensin-converting enzyme 2, angiotensin II (Ang II) receptor subtypes 1 and 2 (AT1R and AT2R) as well as Ang 1-7 receptor (MasR). In the kidneys, Agt is expressed predominantly in renal proximal tubular cells (RPTCs): it is converted into inactive Ang I by renin and then into biologically-active Ang II by ACE. Ang II could be further cleaved to Ang 1-7. Intrarenal RAS gene expression is elevated in diabetes [8], strongly indicating that intrarenal RAS activation plays a significant role in DN progression including interstitial tubulefibrosis and tubular atrophy [9].

Hypertension usually accompanies diabetes mellitus, which increases kidney damage when normo-albuminuria evolves to macroalbuminuria [10]. Ang II, the main product of RAS activation, contributes to various physiological and pathological renal and cardiovascular mechanisms through AT1R stimulation [11]. It has been reported that early streptozotocin (STZ)-induced diabetes downregulates rat kidney AT2R and increases AT1R [12]. Moreover, several studies have shown that AT2R expression is augmented by insulin treatment [13] and down-regulated by Ang II infusion or epidermal 
growth factor treatment [14]. Arresting the effects of RAS activation with either ACE inhibitors [15] to decrease Ang II production or with angiotensin-receptor blockers (ARBs) to block AT1R activation, leads to lower intraglomerular pressure [16], reduced systemic hypertension and, consequently, decreased renal interstitial fibrosis.

\section{Reactive Oxygen Species (ROS)}

Living organisms produce ROS as a result of normal cellular metabolism. ROS steady-state levels are required for cell proliferation, differentiation [17] and degradation of misfolded/damaged proteins by ubiquitin and $26 \mathrm{~S}$ proteasome [18]. In contrast, excessive ROS production damages cellular components, such as DNA, proteins and lipids [19]. Superoxide (O2•-), hydrogen peroxide (H2O2) and nitric oxide are free radicals essential for normal physiological development, but also mediate cellular damage in disease states [20]. Cellular sources of ROS production include plasma membrane nicotinamide adenine dinucleotide phosphate oxidase (NADPH oxidase), intracellular cytosolic xanthine oxidase, peroxisomal oxidases, endoplasmic reticular oxidases and mitochondrial electron transport constituents [19]. Mitochondrial electron transport of aerobic respiration, considered to be the main source of ROS, has been implicated in many disorders $[21,22]$. It has been estimated that 0.2 to $2 \%$ of the oxygen consumed by mitochondria is reduced to $\mathrm{O} 2 \bullet-[23]$.

Oxidative stress occurs in cellular systems when the production of free radical moieties exceeds the antioxidant capacity of those systems, evoking a shift in balance between oxidants/antioxidants in favour of oxidants [24]. In certain pathological conditions, increased generation of ROS and/or antioxidant defence system depletion generate enhanced ROS activity and oxidative stress, resulting in tissue damage. Several systemic diseases, such as hypertension, diabetes mellitus, metabolic syndrome and infections, induce renal oxidative stress [25]. Excessive ROS production in the kidneys has been reported in different hypertensive animal models [26,27], including Ang IIinduced hypertensive rats [28], N-omega-nitro-L-arginine-induced hypertensive rats [29], Dahl salt-sensitive hypertensive rats [30] and spontaneously hypertensive rats [31]

\section{Nrf2 Activation and Redox Balance}

Oxidative stress is the most common cause of tissue injury. In order to maintain redox homeostasis balance to prevent cellular damage, an intracellular antioxidant system, i.e., Nuclear factor erythroid 2-related factor 1-3 (Nrf 1-3), is activated to detoxify the potential harmful substance. Nrf1, Nrf2 and Nrf3 genes encode a member of the cap 'n' collar basic-region leucine zipper family, which is vital in regulating antioxidant gene expression [32], development and redox balance [33]. $\mathrm{Nrf1}$ is an important player in redox balance during development [34]. Homozygous Nrf1 null mice die in utero [35], while Nrf2- and Nrf3null mice develop normally with no obvious phenotypic differences compared to wild type controls [36,37]. Nrf2-deficient female mice develop lupus-like autoimmune nephritis [38]. Nrf1 and Nrf2 deficiency, however, has been shown to culminate in early embryonic lethality and severe oxidative stress [39]. Nrf2 is a transcriptional factor consisting six evolutionarily highly conserved domains, Neh1-6 [40] that activates the transcription of an array of antioxidant genes [41]. Several reports showed that Nrf2 activation may protect against human disease such as cardiovascular disease [42], cancer [43], neurodegenerative diseases [44] and chronic kidney disease [45].

\section{Regulation of Nrf2 activation}

Nrf2 activation is predominantly regulated by it cytosolic partner, Nrf2 adaptor or Kelch-like ECH-associated protein 1 (Keap1). Keap1 is a protein of five domains: two protein-protein interaction motifs, the Kelch domain, the intervening region (IVR), and the BTB domain [46]. Keap1 acts as a cytosolic repressor for Nrf2 in the cytoplasm. Several models have been proposed to understand the mechanism of Nrf2 activation. Under normal physiological conditions, the BTB domain of Keap1 interacts with the Neh2 domain of cytoplasmic Nrf2 and forms complexes with Cullin 3-based E3 ligase [47]. These complexes promote Nrf2 degradation via the ubiquitin proteasome system [48]. In response to oxidative stress or chemo-preventive compounds, Nrf2 dissociates from Keap1 and translocates to the nucleus [49]. However, if Keap 1 binds to other proteins, Nrf2 does not ubiquitinated but activated in a non-canonical, cysteineindependent manner. For example, Lau et al. [50] demonstrated that deregulation of autophagy pathway causes the accumulation of p62 (an autophagic protein) that directly interacts with Keap1, resulting in the inhibition of Keap1-mediated Nrf2 ubiquitination. In addition, Nrf2 has been shown to be regulated at transcriptional level, independent of the Keap1 mechanism. Studies by Kawak et al [51] demonstrated that Nrf2 undergoes autoregulation: Nrf2 binds to its own promoter and induce its own transcriptional activity. Posttranslation modifications also play a significant role in Nrf2 function and localization. For example, Nrf2 phosphorylation by protein kinase $\mathrm{C}$ at serine 40 residue is critical signaling event to regulate cellular antioxidant response [52]. Furthermore, acetylation-deacetylation of the Nrf2 regulates its transcriptional activity and nucleocytoplasmic localization [53]. Natural or synthetic chemopreventive agents, such as curcumin, flavonoids, oltipraz, butylatedhydroxyanisole, and bardoxolone methyl stimulate Nrf2 activation and nuclear translocation. Within the nucleus, Nrf2 forms a heterodimer with Maf protein and bind to regulatory sequences in the promoter region of various genes, known as antioxidant response elements (AREs). A series of antioxidant and cellular protective genes containing AREs in their promoters have been identified; such as glutathione peroxidase, superoxide dismutase, catalase (Cat), heme-oxygenase, NADPHquinoneoxidoreductase 1 and glutamate-cysteine ligase [54,55]. Moreover, it has been found that activation of Nrf2 signaling attenuates NFkappaB-inflammatory response and elicits apoptosis [56].

Recent studies, however, revealed that Nrf2 may also affect noneoxidant genes expression. For example, Pendyala et al. [57] demonstrated in human lung endothelium, by chromatinimmunoprecipitation assay, that $\mathrm{Nrf} 2$ binds to ARE regions in NADPH oxidase-4 (Nox4) gene promoter and up-regulates its activity. NADPH oxidase-1, -2 and -4 (Nox1, Nox2 and Nox4) have also been shown to be expressed in the renal cortex and Nox4 is the most common isoform expressed in the kidneys [58,59]. Nox4 contributes to basal ROS production through its constitutive activity and increases ROS generation when stimulated by Ang II, glucose, and growth factors [60-62]. Our study in the renal proximal tubules (RPTCs) of diabetic Akita mice (a murine model of T1D) has disclosed that Nrf2 translocates to the nucleus with markedly enhanced NADPH oxidase activity as well as Nox4 mRNA expression compared to non-Akita mice, suggesting that Nrf2 induction of Nox4 expression and activity might be responsible, at least in part, for elevated ROS levels [63]. Intriguingly, these changes are normalized by overexpressing catalase (Cat) in RPTCs of Akita mice, lessening Nrf2 translocation to the nucleus [63]. These observations indicated that constitutive Nrf2 
accumulation does not necessary induce absolute protection from environmental influences. Indeed, study has shown that hyperoxia stimulates Nrf2 translocation to the nucleus, and Nrf2 knockdown of Nrf2 gene expression by small interfering RNA (siRNA) attenuates hyperoxia-induced Nox4 expression, suggesting that Nrf2 may upregulates Nox4 gene expression in certain physiological conditions [57].

\section{Nrf2 expression in diabetic kidneys}

Many studies demonstrated a beneficial effect of Nrf2 activation in none-diabetic kidney. Shelton et al. [64] reported that Nrf2 activation protects the kidney against oxidative stress that results from ischemicreperfusion $[65,66]$, renal injury caused by excessive heavy metals $[67,68]$, and ameliorate cyclosporine A nephrotoxicity [69].

In animals with streptozotocin (STZ)-induced diabetes, Nrf2 ablation appears to worsen inflammation, oxidative stress, and nephropathy [70,71]. Moreover, dietary supplementation with sulforaphane or cinnamic aldehyde ( $\mathrm{Nrf} 2$ activators) reduces albuminuria and renal oxidative damage in STZ-induced diabetic mice $[71,72]$. It also improves glucose control, lowers plasma triglyceride and FFA levels, decreases hepatic lipid accumulation and inflammation in both high-fat diet-induced and genetic mouse models of obesity and diabetes [73,74]. Thus, Nrf2 appears to be cytoprotective in none-diabetic and diabetic animal models.

\section{Nrf2 activation in human chronic kidney disease (CKD)}

A phase II trial of bardoxolone methyl (a Nrf2 activator) involving patients with advanced CKD and diabetes was carried out based on compelling evidence of the association between oxidative stress, inflammation and DN progression. Pergola et al. [75] initiated a small clinical trial of 227 T2D patients with moderate to severe CKD (BEAM study) and observed that bardoxolone methyl treatment increased estimated GFR in a dose-dependent manner in patients at 24 weeks with persistence up to 52 weeks. These encouraging data motivated de Zeeuw et al. [76] to investigate the effect of bardoxolone methyl in a phase III clinical trial in 2,185 patients from different countries with stage III CKD and T2D (BEACON Study). However, the trial was terminated early in October 2012 because bardoxolone methyl treatment neither improved renal function nor reduced the risk of ESRD, but was actually associated with increased risk of cardiovascular death. In fact, bardoxolone methyl-treated patients presented significant increment of estimated GFR, blood pressure (BP) and urinary albumin-to-creatinine ratio, with decreased body weight in comparison to the placebo group, and acquired a higher risk of cardiovascular events.

Studies were performed in Zucker diabetic fatty (ZDF) rats, a T2D model, to confirm the potential effects of bardoxolone methyl in DN. Zoja et al. [77] reported that ZDF rats receiving the bardoxolone methyl analogue RTA 405 presented DN deterioration impacting the liver. In contrast, a study of RTA 405 and dh404 sponsored by Reata Pharmaceuticals via Biomodels LLC in ZDF rats showed heightened urinary albumin/creatinine ratios with both compounds, but no adverse effects in the liver [78]. Inconsistencies between these two studies were observed. Subsequent analysis by Reata Pharmaceuticals revealed the presence of unknown impurities in RTA 405 that they had supplied for both studies. The concentrations of these impurities were very low, indicating that they might have been extremely toxic.
Unfortunately, no further work on 'pure' RTA 405 has been reported. Whether Nrf2 activation worsens human CKD progression remains uncertain. Despite such conflicting results, there is still evidence that Nrf2-dependent events are protective against $\mathrm{DN}$ progression $[70,71,73,74]$. These findings led us to investigate whether Nrf2 activator treatment would have adverse side-effects in the kidneys by itself or whether Nrf2 pathway activation would elevate BP and cause kidney injury. To answer this question, we explored the relationship between renal Nrf2 activation and Agt gene expression in the RPTs of wild type mice since we previously reported that high glucose stimulated Agt gene expression via ROS generation [79] and that transgenic mice overexpressing Agt in their RPTCs exhibited hypertension, albuminuria and renal injury [80]. Indeed, we found that oltipraz (a Nrf2 activator) administration heightened both Nrf2 and renal Agt gene expression. Moreover, deletion of Nrf2-response element (RE) sites in the Agt promoter prevented oltipraz stimulation of Agt gene transcription in RPTCs [63]. These observations strongly suggest that $\mathrm{Nrf} 2$ may play dual roles, with stimulation of both antioxidant and hypertensinogenic genes, such as Agt, as illustrated in the schematic Figure 1 below:

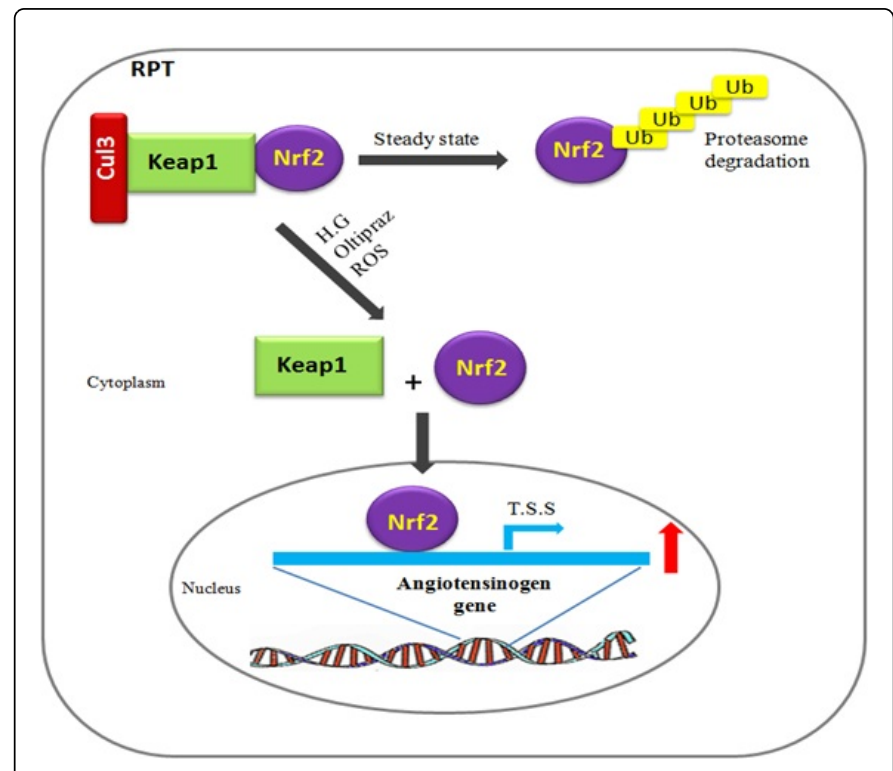

Figure 1: Proposed mechanism of Nrf2 activation on Agt gene expression in RPTs: Under normal conditions, Nrf2 is bound to Keap1 in the cytoplasm and is subject to ubiquitination and proteasome degradation. Upon exposure to high glucose (HG), oxidative stressor or Nrf2 activators (oltipraz), Nrf2-Keap1 complexes dissociate and Nrf2 translocates to the nucleus. Nrf2 binds to Nrf2-REs in the Agt gene promoter, stimulating Agt gene transcription and intrarenal RAS activation, and leading to the development of hypertension and nephropathy in diabetes. TSS (transcriptional start site)

In conclusion, clinical studies have shown that Nrf2 activation by bardoxolonemethy increases systemic hypertension aggravates urinary albumin excretion and augments the risk of cardiovascular death in type II diabetic patients with advanced CKD [76]. These observations suggest that Nrf2 over-activation may not always exhibit beneficial in diabetes. Hyperglycemia-induction of ROS generation and chronic activation of Nrf2 signaling may induce intrarenal RAS activation, lending to systemic hypertension, albuminuria and tubular apoptosis 
and atrophy, further aggravate nephropathy progression. Thus, blocking chronic Nrf2 activation in diabetic kidneys would be a novel therapeutic target for DN treatment. Indeed, the recent study by Tan et al [81] demonstrated that low doses of dh404 lessened and high doses worsened diabetes-associated atherosclerosis and kidney disease in STZ-induced diabetic apoE-/- mice as well as the study by Vaziri et al. [82] demonstrated that in diabetic obese Zucker rats, low doses of dh404 restore Nrf2 activity and ameliorate kidney injury whereas high doses of dh404 reinforce proteinuria, renal dysfunction and histological abnormalities. These observations strongly suggest the possible dual function of Nrf2 activation in the diabetic kidney, depending on the level of $\mathrm{Nrf} 2$ activation in diabetes.

\section{Acknowledgements}

This work was supported by grants from the Canadian Institutes of Health Research (CIHR, MOP 93650 and MOP 16088 to J.S.D.C. and MOP-86450 to S.L.Z.). Editorial assistance was provided by the CRCHUM Research Support Office and Ovid M. Da Silva. No potential conflicts of interest relevant to this article were reported.

\section{Disclosure}

None declared.

\section{References}

1. Guariguata L, Whiting DR, Hambleton I, Beagley J, Linnenkamp U, et al. (2014) Global estimates of diabetes prevalence for 2013 and projections for 2035. Diabetes Res Clin Pract 103: 137-149.

2. Van Buren PN, Toto R (2011) Hypertension in diabetic nephropathy: epidemiology, mechanisms, and management. Adv Chronic Kidney Dis 18: $28-41$.

3. Reutens AT, Atkins RC (2011) Epidemiology of diabetic nephropathy. Contrib Nephrol 170: 1-7.

4. Fonseca VA, Kulkarni KD (2008) Management of type 2 diabetes: oral agents, insulin, and injectables. J Am Diet Assoc 108: S29-33.

5. Hayes C, Kriska A (2008) Role of physical activity in diabetes management and prevention. J Am Diet Assoc 108: S19-23.

6. Johnston CI (1992) Franz Volhard Lecture. Renin-angiotensin system: a dual tissue and hormonal system for cardiovascular control. J Hypertens Suppl 10: S13-26.

7. Kobori H, Nangaku M, Navar LG, Nishiyama A (2007) The intrarenal renin-angiotensin system: from physiology to the pathobiology of hypertension and kidney disease. Pharmacol Rev 59: 251-287.

8. Lai KN, Leung JC, Lai KB, To WY, Yeung VT, et al. (1998) Gene expression of the renin-angiotensin system in human kidney. J Hypertens 16: 91-102.

9. Chawla T, Sharma D, Singh A (2010) Role of the renin angiotensin system in diabetic nephropathy. World J Diabetes 1: 141-145.

10. Perkins BA, Ficociello LH, Ostrander BE, Silva KH, Weinberg J, et al. (2007) Microalbuminuria and the risk for early progressive renal function decline in type 1 diabetes. J Am Soc Nephrol 18: 1353-1361.

11. Siragy HM (2010) The angiotensin II type 2 receptor and the kidney. J Renin Angiotensin Aldosterone Syst 11: 33-36.

12. Wehbi GJ, Zimpelmann J, Carey RM, Levine DZ, Burns KD (2001) Early streptozotocin-diabetes mellitus downregulates rat kidney AT2 receptors. Am J Physiol Renal Physiol 280: F254-265.

13. Samuelsson AM, Bollano E, Mobini R, Larsson BM, Omerovic E, et al (2006) Hyperinsulinemia: effect on cardiac mass/function, angiotensin II receptor expression, and insulin signaling pathways. Am J Physiol Heart Circ Physiol 291: H787-796.

14. Matsubara H (1998) Pathophysiological role of angiotensin II type 2 receptor in cardiovascular and renal diseases. Circ Res 83: 1182-1191.
15. Lewis E, Hunsicker LG, Bain RP, Rohde RD (1993) The effect of angiotensin-converting-enzyme inhibition on diabetic nephropathy. The Collaborative Study Group. N Engl J Med 329: 1456-1462.

16. Sarafidis PA, Stafylas PC, Kanaki AI, Lasaridis AN (2008) Effects of renin-angiotensin system blockers on renal outcomes and all-cause mortality in patients with diabetic nephropathy: an updated metaanalysis. Am J Hypertens 21: 922-929.

17. Chung JS, Lee SB, Park SH, Kang ST, Na AR, et al. (2009) Mitochondrial reactive oxygen species originating from Romol exert an important role in normal cell cycle progression by regulating p27(Kip1) expression. Free Radic Res 43: 729-737.

18. Trachootham D, Lu W, Ogasawara MA, Nilsa RD, Huang P (2008) Redox regulation of cell survival. Antioxid Redox Signal 10: 1343-1374.

19. Valko, M (2007) Free radicals and antioxidants in normal physiological functions and human disease. Int J Biochem Cell Biol,. 39: 44-84.

20. Maxwell SR, Lip GY (1997) Free radicals and antioxidants in cardiovascular disease. Br J Clin Pharmacol 44: 307-317.

21. Turrens JF (2003) Mitochondrial formation of reactive oxygen species. J Physiol 552: 335-344.

22. Musatov A, Robinson NC (2012) Susceptibility of mitochondrial electron-transport complexes to oxidative damage. Focus on cytochrome c oxidase. Free Radic Res 46: 1313-1326.

23. Madamanchi NR, Runge MS (2007) Mitochondrial dysfunction in atherosclerosis. Circ Res 100: 460-473.

24. Birben E, Sahiner UM, Sackesen C, Erzurum S, Kalayci O (2012) Oxidative stress and antioxidant defense. World Allergy Organ J 5: 9-19.

25. Ozbek E (2012) Induction of oxidative stress in kidney. Int J Nephrol 2012: 465897 .

26. Wilcox CS (2002) Reactive oxygen species: roles in blood pressure and kidney function. CurrHypertens Rep 4: 160-166.

27. Nistala R, Whaley-Connell A, Sowers JR (2008) Redox control of renal function and hypertension. Antioxid Redox Signal 10: 2047-2089.

28. Haugen EN, Croatt AJ, Nath KA (2000) Angiotensin II induces renal oxidant stress in vivo and heme oxygenase-1 in vivo and in vitro. Kidney Int 58: 144-152.

29. Rauchova H (2005) Chronic N-acetylcysteine administration prevents development of hypertension in $\mathrm{N}$ (omega)-nitro-L-arginine methyl ester-treated rats: the role of reactive oxygen species. Hypertens Res 28:475-82.

30. Nishiyama A, Yoshizumi M, Hitomi H, Kagami S, Kondo S, et al. (2004) The SOD mimetic tempol ameliorates glomerular injury and reduces mitogen-activated protein kinase activity in Dahl salt-sensitive rats. J Am Soc Nephrol 15: 306-315.

31. Ohashi N, Katsurada A, Miyata K, Satou R, Saito T, et al. (2009) Role of activated intrarenal reactive oxygen species and renin-angiotensin system in IgA nephropathy model mice. Clin Exp Pharmacol Physiol 36: 750-755.

32. McMahon M (2001) The Cap'n'Collar basic leucine zipper transcription factor Nrf2 (NF-E2 p45-related factor 2) controls both constitutive and inducible expression of intestinal detoxification and glutathione biosynthetic enzymes. Cancer Res 61: 3299-307.

33. Schultz MA, AB Abdel, MageedDMondal (2010) The nrf1 and nrf2 balance in oxidative stress regulation and androgen signaling in prostate cancer cells. Cancers (Basel),. 2: 1354-78.

34. Chen L, Kwong M, Lu R, Ginzinger D, Lee C, et al. (2003) Nrf1 is critical for redox balance and survival of liver cells during development. Mol Cell Biol 23: 4673-4686.

35. Chan JY, Kwong M, Lu R, Chang J, Wang B, et al. (1998) Targeted disruption of the ubiquitous CNC-bZIP transcription factor, Nrf- 1 , results in anemia and embryonic lethality in mice. EMBO J 17: 1779-1787.

36. Derjuga A, Gourley TS, Holm TM, Heng HH, Shivdasani RA, et al. (2004) Complexity of CNC transcription factors as revealed by gene targeting of the Nrf3 locus. Mol Cell Biol 24: 3286-3294. 
37. Chan K, Lu R, Chang JC, Kan YW (1996) NRF2, a member of the NFE2 family of transcription factors, is not essential for murine erythropoiesis, growth, and development. Proc Natl Acad Sci U S A 93: 13943-13948.

38. Yoh K, Itoh K, Enomoto A, Hirayama A, Yamaguchi N, et al. (2001) Nrf2-deficient female mice develop lupus-like autoimmune nephritis. Kidney Int 60: 1343-1353.

39. Leung L, Kwong M, Hou S, Lee C, Chan JY (2003) Deficiency of the Nrf1 and Nrf2 transcription factors results in early embryonic lethality and severe oxidative stress. J Biol Chem 278: 48021-48029.

40. Itoh K, Wakabayashi N, Katoh Y, Ishii T, Igarashi K, et al. (1999) Keap1 represses nuclear activation of antioxidant responsive elements by $\mathrm{Nrf} 2$ through binding to the amino-terminal Neh2 domain. Genes Dev 13: 76-86.

41. Li N (2004) Nrf2 is a key transcription factor that regulates antioxidant defense in macrophages and epithelial cells: protecting against the proinflammatory and oxidizing effects of diesel exhaust chemicals. J Immunol 173: 3467-81.

42. Howden R, (2013) Nrf2 and cardiovascular defense. Oxid Med Cell Longev, 2013.: p. 104308.

43. Hong DS, Kurzrock R, Supko JG, He X, Naing A, et al. (2012) A phase I first-in-human trial of bardoxolone methyl in patients with advanced solid tumors and lymphomas. Clin Cancer Res 18: 3396-3406.

44. Yamazaki H, Tanji K, Wakabayashi K, Matsuura S, Itoh K (2015) Role of the Keap1/Nrf2 pathway in neurodegenerative diseases. PatholInt 65: 210-219.

45. Ruiz S, Pergola PE, Zager RA, Vaziri ND (2013) Targeting the transcription factor Nrf2 to ameliorate oxidative stress and inflammation in chronic kidney disease. Kidney Int 83: 1029-1041.

46. Zhang DD, Hannink M (2003) Distinct cysteine residues in Keap1 are required for Keap1-dependent ubiquitination of Nrf2 and for stabilization of Nrf2 by chemopreventive agents and oxidative stress. Mol Cell Biol 23: 8137-8151.

47. Cullinan SB, Gordan JD, Jin J, Harper JW, Diehl JA (2004) The Keap1BTB protein is an adaptor that bridges Nrf2 to a Cul3-based E3 ligase: oxidative stress sensing by a Cul3-Keap1 ligase. Mol Cell Biol 24: 8477-8486.

48. Kobayashi A, Kang MI, Okawa H, Ohtsuji M, Zenke Y, et al. (2004) Oxidative stress sensor Keap1 functions as an adaptor for Cul3-based E3 ligase to regulate proteasomal degradation of Nrf2. Mol Cell Biol 24: 7130-7139.

49. Kobayashi A, Kang MI, Watai Y, Tong KI, Shibata T, et al. (2006) Oxidative and electrophilic stresses activate Nrf2 through inhibition of ubiquitination activity of Keap1. Mol Cell Biol 26: 221-229.

50. Lau A, Wang XJ, Zhao F, Villeneuve NF, Wu T, et al. (2010) A noncanonical mechanism of Nrf2 activation by autophagy deficiency: direct interaction between Keap1 and p62. Mol Cell Biol 30: 3275-3285.

51. Kwak MK, Itoh K, Yamamoto M, Kensler TW (2002) Enhanced expression of the transcription factor Nrf2 by cancer chemopreventive agents: role of antioxidant response element-like sequences in the nrf2 promoter. Mol Cell Biol 22: 2883-2892.

52. Huang HC, Nguyen T, Pickett CB (2002) Phosphorylation of Nrf2 at Ser- 40 by protein kinase $C$ regulates antioxidant response elementmediated transcription. J Biol Chem 277: 42769-42774.

53. Kawai Y (2011) Acetylation-deacetylation of the transcription factor Nrf2 (nuclear factor erythroid 2-related factor 2) regulates its transcriptional activity and nucleocytoplasmic localization. J Biol Chem 286: 7629-40.

54. Jaiswal AK (2004) Nrf2 signaling in coordinated activation of antioxidant gene expression. Free Radic Biol Med 36: 1199-1207.

55. Surh YJ, Kundu JK, Na HK (2008) Nrf2 as a master redox switch in turning on the cellular signaling involved in the induction of cytoprotective genes by some chemopreventive phytochemicals. Planta Med 74: 1526-39.

56. Li W, Khor TO, Xu C, Shen G, Jeong WS, et al. (2008) Activation of Nrf2-antioxidant signaling attenuates NFkappaB-inflammatory response and elicits apoptosis. Biochem Pharmacol 76: 1485-1489.
57. Pendyala S, Moitra J, Kalari S, Kleeberger SR, Zhao Y, et al. (2011) Nrf2 regulates hyperoxia-induced Nox4 expression in human lung endothelium: identification of functional antioxidant response elements on the Nox4 promoter. Free Radic Biol Med 50: 1749-1759.

58. NlanduKhodo S, Dizin E, Sossauer G, Szanto I, Martin PY, et al. (2012) NADPH-oxidase 4 protects against kidney fibrosis during chronic renal injury. J Am Soc Nephrol 23: 1967-1976.

59. Sedeek M, Nasrallah R, Touyz RM, Hébert RL (2013) NADPH oxidases, reactive oxygen species, and the kidney: friend and foe. J Am SocNephrol 24: 1512-1518.

60. Chabrashvili T, Kitiyakara C, Blau J, Karber A, Aslam S, et al. (2003) Effects of ANG II type 1 and 2 receptors on oxidative stress, renal NADPH oxidase, and SOD expression. Am J Physiol RegulIntegr Comp Physiol 285: R117-124.

61. Kim SM, Kim YG, Jeong KH, Lee SH, Lee TW, et al. (2012) Angiotensin II-induced mitochondrial Nox4 is a major endogenous source of oxidative stress in kidney tubular cells. PLoS One 7: e39739.

62. Xi G (2012) Hyperglycemia enhances IGF-I-stimulated Src activation via increasing Nox4-derived reactive oxygen species in a PKCzeta-dependent manner in vascular smooth muscle cells. Diabetes 6: 104-13.

63. Abdo S (2014) Catalase overexpression prevents nuclear factor erythroid 2-related factor 2 stimulation of renal angiotensinogen gene expression, hypertension, and kidney injury in diabetic mice. Diabetes 63: 3483-96.

64. Shelton LM, Park BK, Copple IM (2013) Role of Nrf2 in protection against acute kidney injury. Kidney Int 84: 1090-1095.

65. Liu M, Grigoryev DN, Crow MT, Haas M, Yamamoto M, et al. (2009) Transcription factor Nrf2 is protective during ischemic and nephrotoxic acute kidney injury in mice. Kidney Int 76: 277-285.

66. Wu QQ, Wang Y, Senitko M, Meyer C, Wigley WC, et al. (2011) Bardoxolone methyl (BARD) ameliorates ischemic AKI and increase expression of protective genes Nrf2, PPAR $\hat{I}^{3}$, and HO-1. Am J Physiol Renal Physiol 300: F1180-1192.

67. Calderón J, Ortiz-Pérez D, Yáñez L, Díaz-Barriga F (2003) Human exposure to metals. Pathways of exposure, biomarkers of effect, and host factors. Ecotoxicol Environ Saf 56: 93-103.

68. Chen J, Shaikh ZA (2009) Activation of Nrf2 by cadmium and its role in protection against cadmium-induced apoptosis in rat kidney cells. ToxicolApplPharmacol 241: 81-89.

69. Shin DH, Park HM, Jung KA, Choi HG, Kim JA, et al. (2010) The NRF2heme oxygenase-1 system modulates cyclosporin A-induced epithelialmesenchymal transition and renal fibrosis. Free Radic Biol Med 48: 1051-1063.

70. Jiang T, Huang Z, Lin Y, Zhang Z, Fang D, et al. (2010) The protective role of Nrf2 in streptozotocin-induced diabetic nephropathy. Diabetes 59: 850-860.

71. Zheng H, Whitman SA, Wu W, Wondrak GT, Wong PK, et al. (2011) Therapeutic potential of Nrf2 activators in streptozotocin-induced diabetic nephropathy. Diabetes 60: 3055-3066.

72. Cui W, Bai Y, Miao X, Luo P, Chen Q, et al. (2012) Prevention of diabetic nephropathy by sulforaphane: possible role of Nrf2 upregulation and activation. Oxid Med Cell Longev 2012: 821936.

73. Shin S, Wakabayashi J, Yates MS, Wakabayashi N, Dolan PM, et al. (2009) Role of Nrf2 in prevention of high-fat diet-induced obesity by synthetic triterpenoid CDDO-imidazolide. Eur J Pharmacol 620: 138-144.

74. Saha PK, Reddy VT, Konopleva M, Andreeff M, Chan L (2010) The triterpenoid 2-cyano-3,12-dioxooleana-1,9-dien-28-oic-acid methyl ester has potent anti-diabetic effects in diet-induced diabetic mice and Lepr(db/db) mice. J Biol Chem 285: 40581-40592.

75. Pergola PE, Raskin P, Toto RD, Meyer CJ, Huff JW, et al. (2011) Bardoxolone methyl and kidney function in CKD with type 2 diabetes. N Engl J Med 365: 327-336.

76. de Zeeuw D, Akizawa T, Audhya P, Bakris GL, Chin M, et al. (2013) Bardoxolone methyl in type 2 diabetes and stage 4 chronic kidney disease. N Engl J Med 369: 2492-2503. 
Citation: Abdo S, Zhang SL, Chan JSD (2015) Reactive Oxygen Species and Nuclear Factor Erythroid 2-Related Factor 2 Activation in Diabetic Nephropathy: A Hidden Target. J Diabetes Metab 6: 547. doi:10.4172/2155-6156.1000547

Page 6 of 6

77. ZojaC, Corna D, Nava V, Locatelli M, Abbate M, et al. (2013) Analogs of bardoxolone methyl worsen diabetic nephropathy in rats with additional adverse effects. Am J Physiol Renal Physiol 304: F808-819.

78. Chin M, Lee CY, Chuang JC, Bumeister R, Wigley WC, et al. (2013) Bardoxolone methyl analogs RTA 405 and dh404 are well tolerated and exhibit efficacy in rodent models of Type 2 diabetes and obesity. Am J Physiol Renal Physiol 304: F1438-1446.

79. Hsieh TJ, Zhang SL, Filep JG, Tang SS, Ingelfinger JR, et al. (2002) High glucose stimulates angiotensinogen gene expression via reactive oxygen species generation in rat kidney proximal tubular cells. Endocrinology 143: 2975-2985.
80. Sachetelli S, Liu Q, Zhang SL, Liu F, Hsieh TJ, et al. (2006) RAS blockade decreases blood pressure and proteinuria in transgenic mice overexpressing rat angiotensinogen gene in the kidney. Kidney Int 69: 1016-1023.

81. Tan SM (2014) Derivative of bardoxolone methyl, dh404, in an inverse dose-dependent manner lessens diabetes-associated atherosclerosis and improves diabetic kidney disease. Diabetes 6: 3091-103.

82. Vaziri ND, Liu S, Farzaneh SH, Nazertehrani S, Khazaeli M, et al. (2015) Dose-dependent deleterious and salutary actions of Nrf2 inducer, dh404, in chronic kidney disease. Free Radic Biol Med. 